Check for updates

Cite this: RSC Adv., 2018, 8, 28433

\title{
Preparation and characterization of chitosan membranes
}

\author{
Luqing Cui, (DD a Shanshan Gao, ${ }^{\text {ab }}$ Xiaoming Song, ${ }^{\text {ab }}$ Lianqing Huang, ${ }^{a}$ Hehe Dong, ${ }^{a}$ \\ Jinling Liu, ${ }^{a}$ Fushan Chen ${ }^{a}$ and Shitao $\mathrm{Yu}^{\mathrm{a}}$
}

The present study investigates a new solvent system for the dissolution of chitosan and a new method for preparing chitosan membranes. First, aqueous tartaric acid was used to pretreat chitosan. Then, the chitosan was precipitated with ethanol or other regenerating agents, and $1.5 \mathrm{~mL}$ of 1-ethyl-3methylimidazolium acetate ([EMIM]AC) was added to obtain translucent suspensions. The chitosan membranes were prepared by casting the suspensions on glass plates and allowing solvent evaporation. The structure and properties of the films were investigated by SEM, FT-IR, XRD and TGA. Also, the mechanical properties, as well as physical and chemical characteristics, of the chitosan films were evaluated. The results indicated that the optimum dissolution time was $10 \mathrm{~min}$ and the most suitable drying temperature was $60{ }^{\circ} \mathrm{C}$. The thus-prepared film was moderately thick (about $0.02 \mathrm{~mm}$ ) and had a smooth surface, without curling. The chitosan film prepared by ethanol regeneration had a tensile strength of up to $24 \mathrm{MPa}$, a minimum swelling degree of $78 \%$, and a water vapor transmission rate of $270 \mathrm{~g} \mathrm{~m}^{-2} \mathrm{~d}^{-1}$ without the addition of plasticizer.

Received 28th June 2018 Accepted 30th July 2018

DOI: $10.1039 / c 8 r a 05526 b$

rsc.li/rsc-advances necessary to find an alternative dissolution medium for chitosan. ${ }^{9}$

Tartaric acid is a white crystalline, dibasic organic acid, which has been found to have many applications, for example, as acidifier, antioxidant, flavor enhancer, etc., in the wine, bakery and food industries, as well as in pharmaceutical industries. ${ }^{\mathbf{1 0 - 1 2}}$ The wide use of tartaric acid in various fields due to its excellent properties, including safety, large adsorption capacity, high adsorption efficiency and good biodegradability. ${ }^{13-15}$

Ionic liquids (ILs) are a group of salts that exist in liquid form at relatively low temperatures $\left(<100{ }^{\circ} \mathrm{C}\right)$ and have recently received increasing attention as new environmentally friendly solvents. They have been proved to have suitable chemical and thermal stability. ${ }^{16,17}$ Swatloski et al. ${ }^{18}$ were the first to find that imidazole-based ionic liquids can effectively dissolve cellulose, generating widespread interest. The use of ionic liquids as a dissolution medium for chitosan has also attracted wide research interest, due to the similar structures of chitosan and cellulose. Numerous studies have shown that 1-ethyl-3methylimidazolium acetate is an excellent solvent for chitosan. ${ }^{19}$ However, ionic liquids are highly viscous and difficult to recover, ${ }^{20-22}$ which, in addition to the high dissolution temperature, the low concentration of chitosan dissolved in ionic liquids limits the use of ionic liquids as a single solvent for chitosan.

This study investigates a new solvent system for the dissolution of chitosan and a new method for preparing chitosan membranes. First, aqueous tartaric acid was used to pretreat
${ }^{a}$ Shandong Provincial Key Laboratory of Biochemical Engineering, College of Marine Science and Biological Engineering, Qingdao University of Science \& Technology, Qingdao, Shandong 266042, China.E-mail: ss4007@sina.com

${ }^{b}$ Key Laboratory of Pulp and Paper Science \& Technology of Ministry of Education/ Shandong Province, Qilu University of Technology, Jinan, 250353, China 
chitosan, then ethanol or other regenerating agents were added and finally [EMIM]AC was used to obtain a translucent suspension. The chitosan film is prepared by casting and solvent evaporation, and the residual [EMIM]AC is removed by washing with deionized water, which allows effectively overcoming the defects of chitosan films, such as fragility and curling upon drying at low concentrations of chitosan. At the same time, the properties of the chitosan membranes were analyzed in order to investigate their suitability for packaging applications (water sensitivity, mechanical properties, etc.).

\section{Experimental section}

\subsection{Materials}

Chitosan (degree of deacetylation $=85 \%$ ), purchased from Shanghai Lanji Technology Development Co., Ltd., was vacuum dried at $80{ }^{\circ} \mathrm{C}$ for 10 hours before use. Tartaric acid was purchased from Shanghai EBI Chemical Reagent Co., Ltd. 1Ethyl-3-methylimidazolium acetate was purchased from Shenyang Synox Technology Co., Ltd. and its purity was $90 \%$. It was vacuum-dried at $80{ }^{\circ} \mathrm{C}$ for $24 \mathrm{~h}$ before use. The remaining reagents were of analytical grade.

\subsection{Experimental methods and characterization}

2.2.1 Determination of chitosan molecular weight. The molecular weight of chitosan was determined according to the procedure described by Chen et al. ${ }^{23} \mathrm{~A}$ certain amount of purified chitosan was accurately weighed with a balance, added to a certain amount of solvent $\left(0.1 \mathrm{~mol} \mathrm{~L}^{-1}\right.$ acetic acid solution and $0.2 \mathrm{~mol} \mathrm{~L}^{-1}$ sodium chloride mixed solution) and stirred thoroughly until complete dissolution. Then, $15 \mathrm{~mL}$ of chitosan solution was pipetted into an Ubbelohde viscometer placed in a water bath at $25{ }^{\circ} \mathrm{C}$. After reaching constant temperature, the flow time of the chitosan solution was measured using a stopwatch. After the test was completed, the Mark-Houwink equation was used to calculate the molecular weight: $[\eta]=K M^{\alpha}$, where $K=10^{-30} \times \mathrm{DD},{ }^{14} \alpha=-1.02 \times 10^{-2} \times \mathrm{DD}+1.82$, DD is the degree of deacetylation of chitosan, $M$ is the viscosity average molecular weight of chitosan.

2.2.2 Preparation of chitosan suspension. In order to prepare a $1.5 \%$ tartaric acid solution, $0.4569 \mathrm{~g}$ of tartaric acid and $30 \mathrm{~g}$ of deionized water were added to a four-necked flask and magnetically stirred as the temperature was raised to $60{ }^{\circ} \mathrm{C}$. Then, $0.6091 \mathrm{~g}$ of chitosan was added to the solution and stirring was continued for 15 min until complete dissolution of the chitosan, resulting in a $2.0 \%$ chitosan solution. The chitosan solution was then turned into a flocculent suspension using a regenerating agent such as ethanol and then $1.5 \mathrm{~mL}$ of [EMIM] AC was added to it. The temperature was raised to $70{ }^{\circ} \mathrm{C}$ and stirring was continued for 30 min resulting in a translucent chitosan solution. The procedure was performed for three different regenerating agents: ethanol, acetonitrile, and acetone.

2.2.3 Observation of the dissolution process of chitosan. Chitosans with different dissolution times, of $1 \mathrm{~min}, 3 \mathrm{~min}$, $7 \mathrm{~min}$, and $10 \mathrm{~min}$, as well as the translucent chitosan solutions obtained using different regenerating agents, were observed with a PH100 biomicroscope.

2.2.4 Preparation of the chitosan film. The chitosan films were prepared by the method of casting and evaporation of the solvent. The translucent suspensions of chitosan prepared by the procedure described above were cast on glass plates, dried at $60{ }^{\circ} \mathrm{C}$ for $2.5 \mathrm{~h}$, and the residual [EMIM]AC is removed by washing with deionized water, and then stored in a desiccator until use.

2.2.5 SEM analysis. The surface morphology of the chitosan films prepared with different regenerating agents was observed using a scanning electron microscope, operating at a voltage of $3.5 \mathrm{kV}$. The samples were sputter coated with gold for approximately 90 seconds.

2.2.6 FT-IR analysis. FT-IR spectra of the chitosan films made with different regenerating agents were recorded on a VERTEX 70 Fourier transform infrared spectrometer in the range of $500-4000 \mathrm{~cm}^{-1}$. The samples for FR-IR analysis were prepared by the $\mathrm{KBr}$ pellet method.

2.2.7 XRD analysis. XRD analysis was performed using a PANALYTICAL XPERT-PRO wide angle X-ray diffractometer (The Netherlands). The experimental conditions were as follows: $\mathrm{Cu}$ target, $\mathrm{K} \alpha$ ray (wavelength of $0.154 \mathrm{~nm}$ ), voltage of 40 $\mathrm{kV}$, current of $40 \mathrm{~mA}$, scanning range of $3-80^{\circ}$.

2.2.8 TG analysis. TGA was carried out on a TG209F1 thermogravimetric analyzer (Germany) at a heating rate of $10{ }^{\circ} \mathrm{C} \mathrm{min}{ }^{-1}$ under $\mathrm{N}_{2}$ flow at a flow rate of $20 \mathrm{~mL} \mathrm{~min}{ }^{-1}$, on sample amounts of $5-10 \mathrm{mg}$.

2.2.9 Determination of mechanical properties. The thickness of the chitosan films prepared with different regenerants was measured using a thickness gauge. A standard paper cutting knife was used to cut the films to a length of $40 \mathrm{~mm}$ and a width of $20 \mathrm{~mm}$. The tensile strength of the composite films was measured using a TTM computer-based tensile tester with a $30 \mathrm{~mm}$ spacing, at a tension of $5 \mathrm{~N}$.

Then, the tensile strength was calculated according to the formula: $\sigma=F / b d$, where $F$ is the breaking load, $\mathrm{N} ; b$ is the film width, $\mathrm{mm}$; $d$ is the film thickness, $\mathrm{mm}$.

2.3.0 Swelling. The chitosan films prepared with different regenerants were dried to constant weight (denoted as Go), placed in deionized water and soaked for 24 hours. Then, the films were taken out of the water, quickly wiped with filter paper to remove surface moisture, and then weighed again. The wet weight of the films was recorded as Gt. The degree of swelling was calculated according to the formula: swelling degree (\%) = (Gt - Go)/Go, where Gt is the weight of wet film and Go is the weight of the dry film.

2.3.1 Water vapor permeability. The chitosan films prepared with different regenerants were cut to a size of 20 he20 $\mathrm{mm}$. The test sample was fixed with glue to the opening of a conical flask containing $5 \mathrm{~mL}$ of deionized water. The water vapor permeability was determined at room temperature by measuring the change in the weight of the system. The water vapor transmission rate was calculated by the following formula: $(\mathrm{WVTR})=\left(W_{t}-W_{\mathrm{o}}\right) /(t A)$, where $W_{\mathrm{o}}$ is the mass of the initial system, $W_{t}$ is the mass of the system at time $t, t$ is the measurement time, and $A$ is the open area of the conical flask. ${ }^{24}$ 


\section{Results and discussion}

\subsection{Determination of molecular weight of chitosan}

Table 1

Table 1 Determination of molecular weight of chitosan ${ }^{a}$

\begin{tabular}{lcccc}
\hline $\begin{array}{l}\text { Solution concentration } \\
\left(\mathrm{mg} \mathrm{mL}^{-1}\right)\end{array}$ & Flow time/s & $\eta_{\mathrm{r}}$ & $\eta_{\mathrm{sp}}$ & $\eta_{\mathrm{sp}} / \mathrm{C}$ \\
\hline 0.001 & 154.54 & 1.47 & 0.47 & 465.87 \\
0.00075 & 140.02 & 1.33 & 0.33 & 437.56 \\
0.0006 & 130.34 & 1.24 & 0.24 & 393.97 \\
0.0004 & 120.95 & 1.25 & 0.15 & 368.28 \\
0 & 105.42 & & & \\
\multicolumn{5}{l}{ Calculated from the Mark-Houwink equation: $[\eta]=K M^{\alpha}$ The } \\
molecular weight of chitosan $M=398300$.
\end{tabular}

\subsection{Observation of chitosan dissolution process}

Fig. 1 illustrates that the chitosan completely dissolved in the tartaric acid aqueous solution after $10 \mathrm{~min}$, forming a homogeneous and stable system. Prior to reaching this final state, the chitosan underwent the stages of swelling and partial dissolution. Upon the addition of the regenerating agents large flake-like flocks were formed, after which [EMIM]AC was added. The resulting translucent suspensions of chitosan are shown in Fig. 1(a-c). It can be clearly seen that the flocks are larger in the suspensions obtained by means of acetonitrile and acetone. Larger flocks may lead to their accumulation during the coating process, which then increases the thickness of the dried chitosan films. This affects the mechanical properties of the entire membrane. It can be inferred that the chitosan film prepared with ethanol as a regenerant would have better mechanical properties.

\subsection{SEM and membrane morphology analysis}

Scanning electron microscopy images exhibit the surface morphology of the chitosan film and regenerated chitosan films.
It may be noted in Fig. 2(a1 and b1) that the surface of the chitosan film prepared from the chitosan solution presents impurities, while that of the translucent suspension regenerated with ethanol is smooth and flat, without any cracks. On the other hand, the chitosan suspensions regenerated with acetonitrile and acetone produced larger flocks, which resulted in irregularities on the surface of the chitosan membranes, but while their surfaces are not very smooth, they present no pores. It can be probably explained by the excessive flocculation caused by acetonitrile and acetone, which hindered the rearrangement of the regenerated chitosan molecules during the drying process. Fig. 2(a2 and b2) reveal that the chitosan film prepared by the common solution-casting method tends to curl after drying and is thin and brittle, which is known to be associated with low concentrations of chitosan. Considering the discussion above, it can be concluded that ethanol is a suitable regenerant for the preparation of chitosan membranes, while chitosan membrane formation by suspension is a feasible method.

\subsection{The structure and properties of the films}

Fig. 3 reveals the peak corresponding to the hydroxyl group $(-\mathrm{OH})$ at approximately $3400 \mathrm{~cm}^{-1}$, and that assigned to the multiple stretching vibration formed by the amine group $\left(-\mathrm{NH}_{2}\right)$ at $3000 \mathrm{~cm}^{-1} \cdot{ }^{25}$ This shows that there are strong and weak intramolecular and intermolecular hydrogen bonds between $-\mathrm{OH}$ and $-\mathrm{NH}_{2}$. The peak at $2880 \mathrm{~cm}^{-1}$ belongs to the $\mathrm{CH}$ stretching vibration, and that at $1647 \mathrm{~cm}^{-1}$ is ascribed to the $\mathrm{NH}, \mathrm{CO}(\mathrm{I})$ stretching vibration due to the incomplete deacetylation of chitosan. The peaks at $1078 \mathrm{~cm}^{-1}$ and $1031 \mathrm{~cm}^{-1}$ are assigned to CO stretching vibrations. ${ }^{26}$ All the peaks discussed above belong to the characteristic peaks of chitosan, which allows concluding that the main functional groups of chitosan did not change after its dissolution and regeneration. Also, it can be seen from Fig. 3 (c and d) that the peak at $3400 \mathrm{~cm}^{-1}$ is significantly shifted to the left, and there is less stretching vibration in the range of $1100-1500 \mathrm{~cm}^{-1}$. Fig. $3 \mathrm{~b}$ clearly reveals that the molecular structure of the ethanol-regenerated
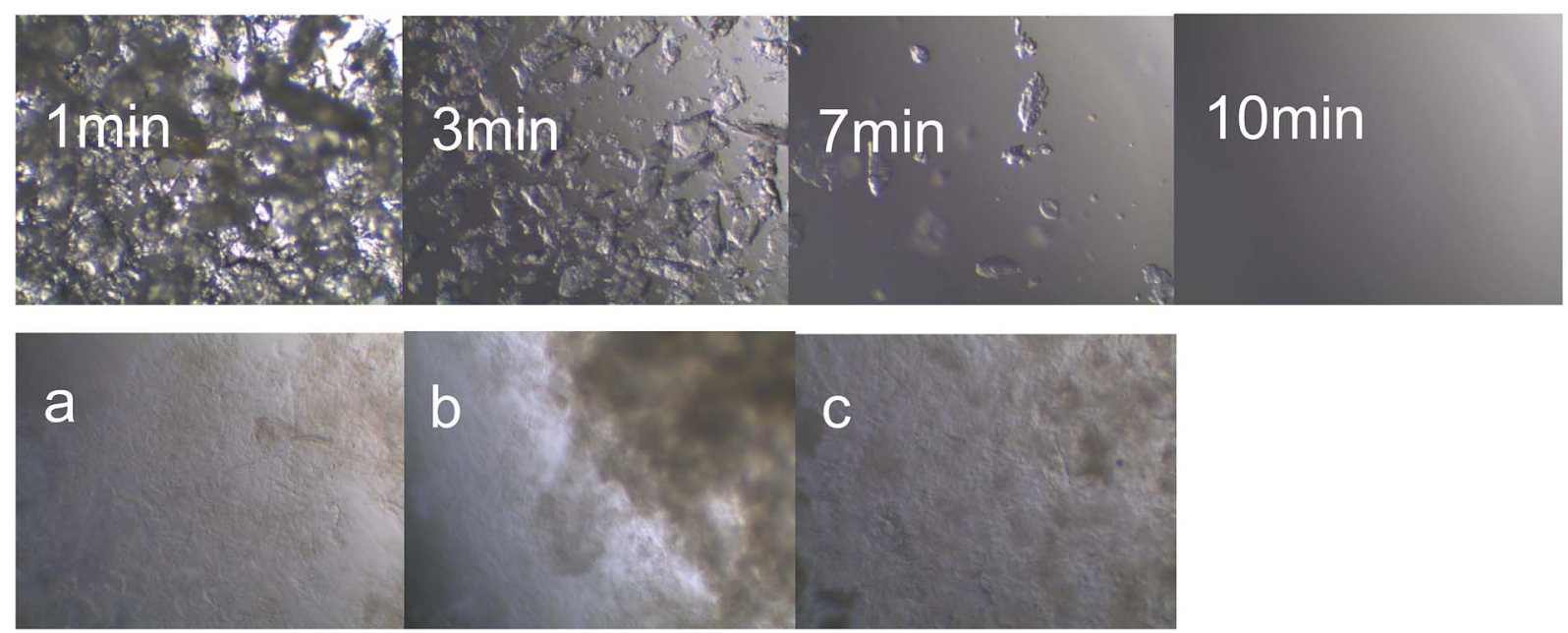

Fig. 1 Microscopic observation of the dissolution and regeneration of the chitosan suspension using (a) ethanol, (b) acetonitrile, and (c) acetone. 


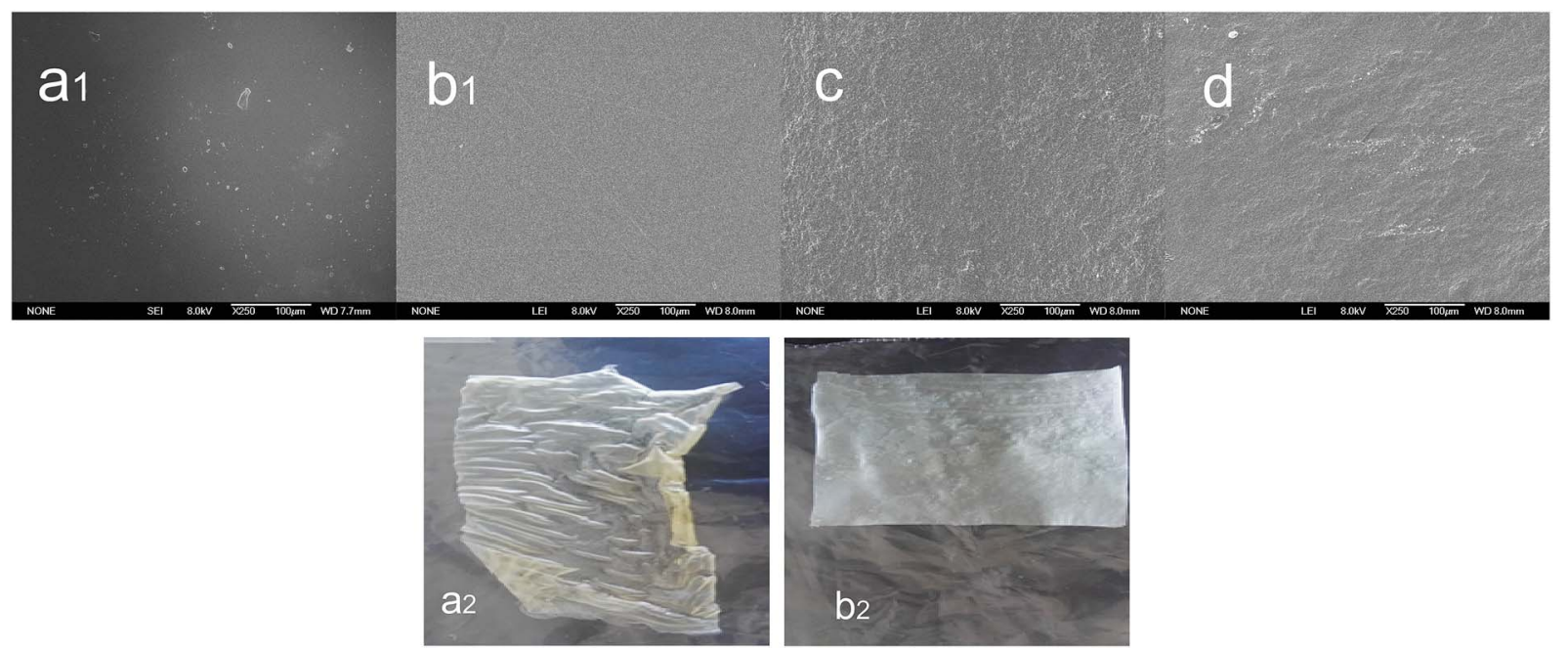

Fig. 2 SEM images of chitosan films prepared from (a) chitosan solution, and chitosan suspension using (b) ethanol, (c) acetonitrile, and (d) acetone.

chitosan is most similar to that of the chitosan film prepared from the chitosan solution. It is possible that during the reconstitution of the regenerated solution, the $-\mathrm{NH}_{2}$ in the ethanol-regenerated chitosan molecule forms a hydrogen bond with the negatively-charged $\mathrm{CH}_{3} \mathrm{COO}-$ in [EMIM]Ac. Acetonitrile and acetone have weak hydrogen bonding because of the large intermolecular steric hindrance.

Fig. 4 shows that the X-ray diffraction patterns of the neat chitosan and of those formed by means of the different regenerating agents. As can be seen from the figure, chitosan has three characteristic absorption peaks at $2 \theta=10.22^{\circ}, 19.9^{\circ}$ and $20.5^{\circ},{ }^{27}$ while the peaks of the ethanol-regenerated chitosan are observed at $2 \theta=10.2^{\circ}, 19.02^{\circ}$ and $21.2^{\circ}$. Thus, the X-ray diffraction profiles of the two films are basically the same, as the intensity of the peaks is also very close. The width of the crystallization peak is slightly increased, indicating that the

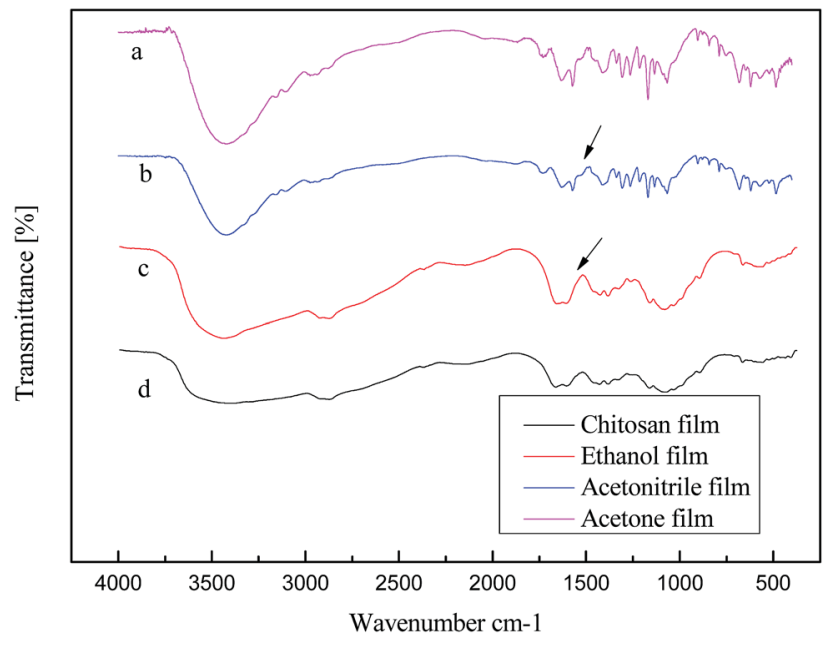

Fig. 3 Infrared spectra of chitosan films prepared from (a) chitosan solution, and chitosan suspension using (b) ethanol, (c) acetonitrile, and (d) acetone. crystal grains became smaller. On the other hand, the corresponding peak near $2 \theta=10^{\circ}$ in the patterns of the acetonitrile and acetone regenerated chitosan films basically disappeared, while the peak strength at $2 \theta=20.6^{\circ}$ obviously decreased, showing loss of crystallinity. In line with the infrared analysis above, the number of the hydrogen bonds formed in the ethanol regenerated chitosan film with the addition of [EMIM]Ac is greater than that of the hydrogen bonds formed in the films prepared with acetonitrile, acetone and [EMIM]Ac, where the latter is loosely arranged among the chitosan molecules, resulting in a significant decrease in the crystalline phase.

Fig. 5 exhibits the TG curves of the chitosan films developed from the solution and from regenerated chitosan suspensions. As can be seen from the figure, the weight loss process of chitosan is divided into two phases. The first stage occurred before $150{ }^{\circ} \mathrm{C}$. The mass loss at this stage was relatively small, ranging from $4 \%$

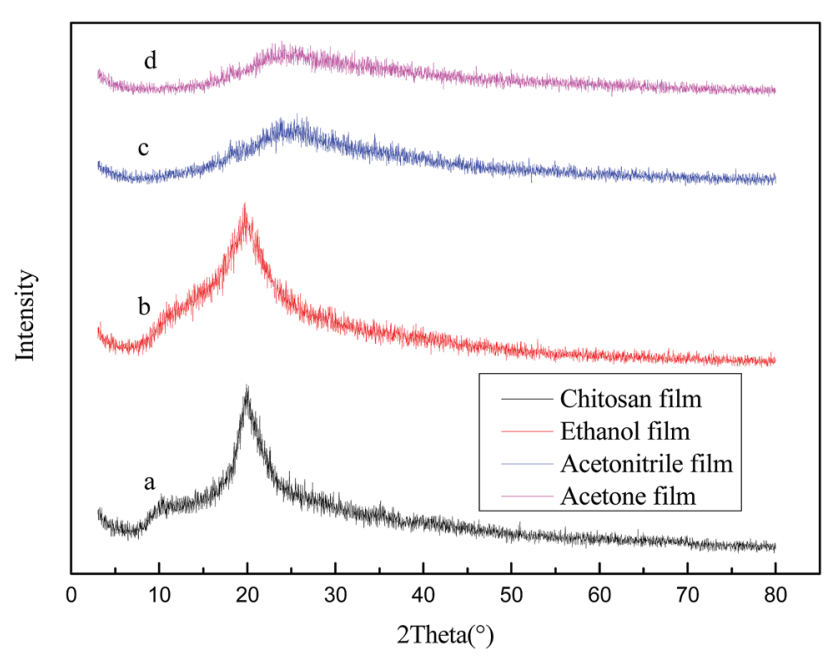

Fig. 4 X-ray diffraction patterns of chitosan films prepared from (a) chitosan solution, and chitosan suspension using (b) ethanol, (c) acetonitrile, and (d) acetone. 


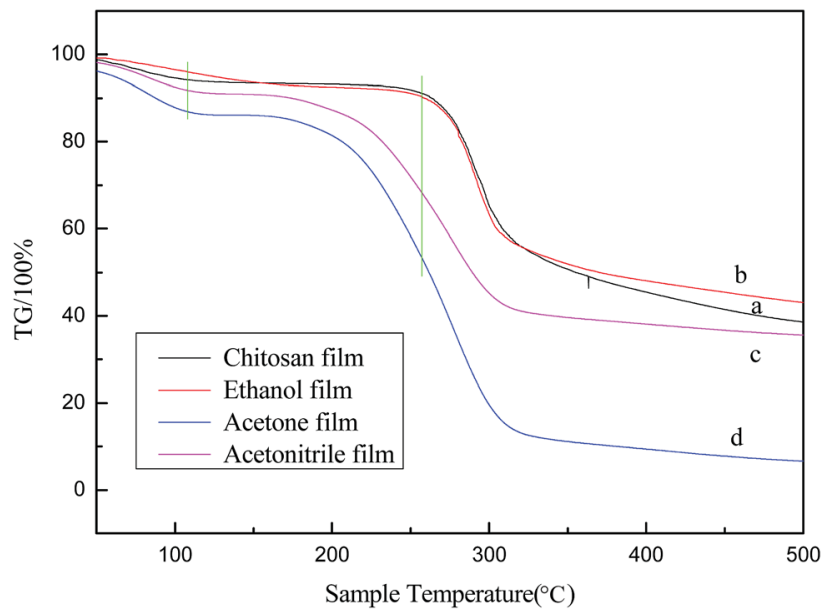

Fig. 5 TG diagrams of chitosan membranes prepared from (a) chitosan solution, and chitosan suspension using (b) ethanol, (c) acetonitrile, and (d) acetone.

to $15 \%$, but the weight loss rate of chitosan molecules regenerated by means of acetonitrile and acetone was greater than in the case of ethanol. The weight loss at this stage is due to water evaporation..$^{28,29}$ The second phase occurs between $180-400{ }^{\circ} \mathrm{C}$, this is because the decomposition of chitosan leads to rapid weight loss. It can be seen from Fig. $5 \mathrm{~d}$ that the weight loss rate of acetone-regenerated chitosan molecules reaches $85 \%$, while that of the ethanol-regenerated film is the lowest. The ethanolregenerated chitosan molecule has the highest decomposition temperature, which is about $260{ }^{\circ} \mathrm{C}$. This may be due to the hydrogen bonds generated that make the intermolecular stability stronger. Considering the analysis above, the explanation may be that the changes in the crystalline state of chitosan molecules cause large differences in thermal stability. Also, these differences may lead to changes in mechanical strength.

\subsection{Determination of mechanical properties and water sensitivity of chitosan membranes}

Mechanical properties are very important for judging the suitability of materials for packaging applications. Among them, tensile strength is an indicator of major significance for evaluating packaging materials, while drying temperature and film formation methods have a great impact on this property.

It can be noted from Fig. 6 that the tensile strength of the films shows an upward trend with increasing drying temperature. As the temperature rises, the solvent evaporates faster, resulting in a rapid rearrangement of the curled chitosan molecular chains, the gap between the molecules is increasingly smaller, and the binding is tighter, so the mechanical strength is improved. It can be also clearly seen from Fig. 6 that the mechanical strength of the membrane prepared from the semi-transparent suspension regenerated with ethanol is significantly higher than those of the other membranes (the film strength prepared by chitosan solution is generally $15-18 \mathrm{MPa}^{30}$ ). In view of the above analysis, it is possible that the flocculated chitosan molecules in the suspension evaporate more rapidly during the drying process, and the formation of intermolecular hydrogen bonds improves the

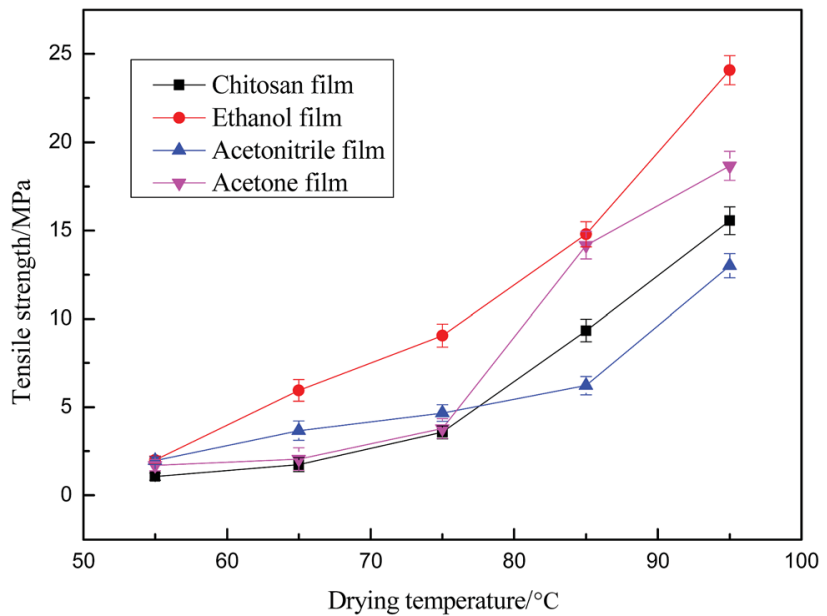

Fig. 6 Effect of drying temperature on tensile strength of membranes prepared from (a) chitosan solution, and chitosan suspension using (b) ethanol, (c) acetonitrile, and (d) acetone.

mechanical properties of the resulting film. On the other hand, in the case of the film prepared by the common solution-casting method, the chitosan molecule is smaller and more fluid, the evaporation rate of the solvent is lower than the diffusion rate of the molecular surface, and as a result, the obtained film tends to be curled and wrinkles are uneven, and therefore its mechanical properties are reduced. Secondary drying of the chitosan film prepared by ordinary solution will cause severe deformation, such as shrinkage and curling, and substantially no mechanical strength, as shown in Fig. 2(a2), while the mechanical strength of the chitosan film prepared by the ethanol regeneration suspension can still reach $24 \mathrm{MPa}$ after the secondary drying, and does not affect the use of the film.

In addition to mechanical properties, the water sensitivity characteristic is also an important indicator for packaging applications. With regard to food packaging films, the requirements for water sensitivity are quite different, depending on the packaged items.

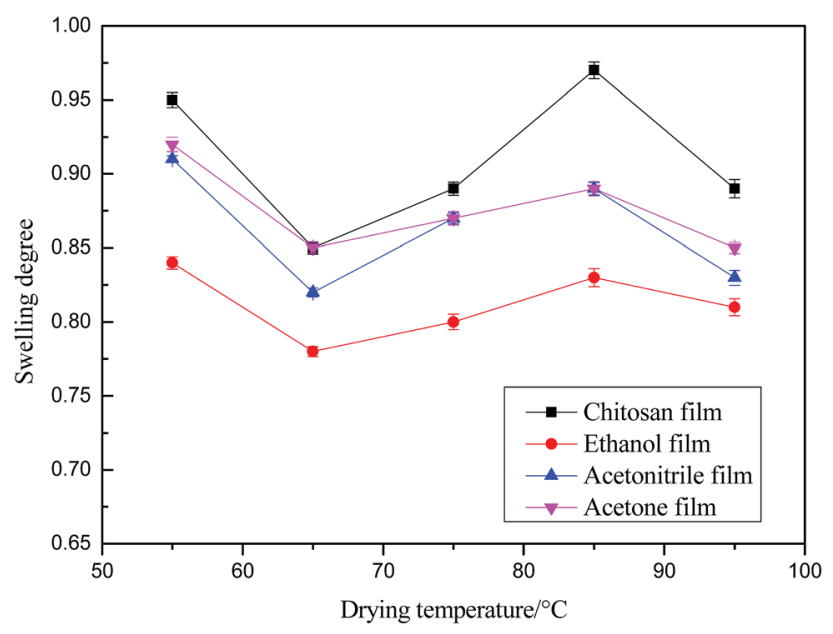

Fig. 7 Effect of drying temperature on the swelling rate of chitosan films prepared from chitosan solution, and chitosan suspension using ethanol, acetonitrile, and acetone. 


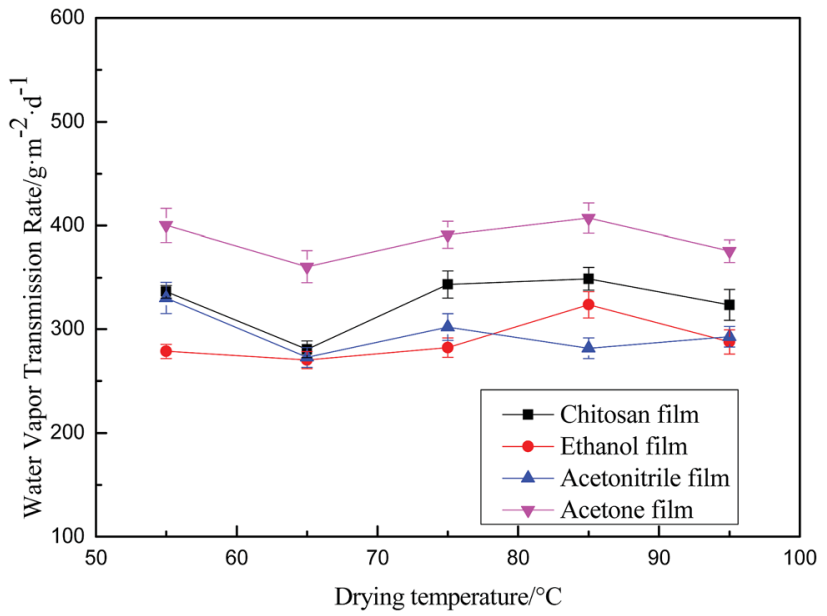

Fig. 8 Effect of drying temperature on water vapor permeability of chitosan membranes prepared from chitosan solution, and chitosan suspension using ethanol, acetonitrile, and acetone.

Fig. 7 and Fig. 8 indicate that the trend of the swellability of the chitosan films is the same as that of the water vapor transmission rate, both showing a tendency of decreasing first and then increasing as a function of drying temperature. The water vapor transmission rate and the swelling degree are the minimum at the drying temperature of $65{ }^{\circ} \mathrm{C}$. The chitosan membrane prepared from the ethanol-regenerated suspension is closest to the chitosan membrane prepared by the solution-casting method in terms of water sensitivity. It has been thus demonstrated that the drying temperature is a major factor that affects the water sensitivity of chitosan membranes. At the same time, the water vapor transmission rate of ethanol regeneration film is the lowest and, in conclusion, this film can be used to package foods, such as fruits and vegetables, which lose moisture easily.

\section{Conclusion}

In this paper, chitosan membranes have been developed and characterized with regard to their properties in order to investigate their suitability for packaging applications. In order to prepare the membranes, aqueous tartaric acid was first used to pretreat chitosan, then the chitosan was precipitated with ethanol or other regenerating agents, and [EMIM]AC was added to obtain translucent suspensions. The chitosan membranes were prepared by the solution casting and solvent evaporation method, which effectively solved the problems associated with the low concentration of chitosan. Lower chitosan concentration has the disadvantage of forming fragile films that curl upon drying. Upon rearrangement of the chitosan molecules, the tensile strength of the resulting chitosan films was greatly increased, without requiring the addition of a plasticizer, such as glycerin. This method has been demonstrated as an excellent for preparing chitosan films.

\section{Conflicts of interest}

There are no conflicts to declare.

\section{Acknowledgements}

This work was supported by the Natural Science Foundation of Shandong Province (ZR2017QB002), one of the key scientific research projects in Shandong Province (2018GGX104003), the Taishan Scholar Program of Shandong (ts201511033), China's post-doctoral funding projects (2018M632638) and Shandong Province major innovation project in 2017 (2018CXGC1001).

\section{References}

1 Y. Wei, J. Wang, H. Li, M. Zhao, H. F. Zhang, Y. P. Guan, H. Huang, B. X. Mi and Y. S. Zhang, Partially reduced graphene oxide and chitosan nanohybrid membranes for selective retention of divalent cations, RSC Adv., 2018, 8(25), 13656-13663.

2 J. Berger, M. Reist, J. M. Mayer, O. Felt, N. A. Peppas and R. Gurny, Structure and interactions in covalently and ionically crosslinked chitosan hydrogels for biomedical applications, Eur. J. Pharm. Biopharm., 2004, 57(1), 19-34.

3 W. A. Neugebauer, E. Neugebauer and R. Brzezinski, Determination of the degree of $\mathrm{N}$-acetylation of chitinchitosan with picric acid, Carbohydr. Res., 1989, 189(89), 363-367.

4 S. S. Silva, J. F. Mano and L. R. Rui, Ionic liquids in the processing and chemical modification of chitin and chitosan for biomedical applications, Green Chem., 2017, 19(5), 1208-1220.

5 C. King, J. Shamshina, G. Gurau, P. Berton, N. F. A. F. Khan and R. D. Rogers, A platform for more sustainable chitin films from an ionic liquid process, Green Chem., 2017, 19(1), 117-126.

6 A. C. Galvis-Sánchez, A. M. M. Sousa, L. Hilliou, M. P. Gonçalves and H. K. S. Souza, Thermo-compression molding of chitosan with deep eutectic mixture for biofilms development, Green Chem., 2016, 18(6), 1571-1580.

7 W. Z. Qiu, Q. Z. Zhong, Y. Du, Y. Lv and Z. K. Xu, Enzymetriggered coatings of tea catechins/chitosan for nanofiltration membranes with high performance, Green Chem., 2016, 18(23), 6205-6208.

8 D. Huang, L. L. Niu, J. Li, J. J. Du, Y. Wei, Y. C. Hu, X. J. Lian, W. Y. Chen and K. Q. Wang, Reinforced chitosan membranes by microspheres for guided bone regeneration, J. Mech. Behav. Biomed. Mater., 2018, 81, 195-201.

9 U. Regmi, M. Palma and C. G. Barroso, Direct determination of organic acids in wine and wine-derived products by Fourier transform infrared (FT-IR) spectroscopy and chemometric techniques, Anal. Chim. Acta, 2012, 732(732), 137-144.

10 Z. J. Ban, B. Horev, R. Rutenberg, O. Danay, C. Bilbao, T. McHugh, V. Rodov and E. Poverenov, Efficient production of fungal chitosan utilizing an advanced freezethawing method; quality and activity studies, Food Hydrocolloids, 2018, 81, 380-388.

11 K. N. Kontogiannopoulos, S. I. Patsios and A. J. Karabelas, Tartaric acid recovery from winery lees using cation 
exchange resin: Optimization by Response Surface Methodology, Sep. Purif. Technol., 2016, 165, 32-41.

12 V. Sasikala, D. Sajan, N. Vijayan, K. Chaitanya, M. S. Babu Raj and B. H. Selin Joy, Growth, molecular structure, NBO analysis and vibrational spectral analysis of l-tartaric acid single crystal, Spectrochim. Acta, Part A, 2014, 123(123C), 127-141.

13 N. Wang, P. R. Chang, P. W. Zheng and X. F. Ma, Carbon nanotube-cyclodextrin adducts for electrochemical recognition of tartaric acid, Diamond Relat. Mater., 2015, 55, 117-122.

14 L. Habala, R. Horáková and R. Čižmáriková, Chromatographic separations based on tartaric acid and its derivatives, Monatsh. Chem., 2018, 149(5), 873-883.

15 Q. Cheng, D. Wang and H. P. Zhou, Electrodeposition of $\mathrm{Zn}(\mathrm{O}, \mathrm{S})$ (zinc oxysulfide) thin films: exploiting its thermodynamic and kinetic processes with incorporation of tartaric acid, J. Energy Chem., 2018, 27(3), 913-922.

16 B. S. Wang, L. Qin, T. C. Mu, Z. M. Xue and G. H. Gao, Are Ionic Liquids Chemically Stable?, Chem. Rev., 2017, 117(10), 7113-7131.

17 Y. Y. Cao and T. C. Mu, Comprehensive Investigation on the Thermal Stability of 66 Ionic Liquids by Thermogravimetric Analysis, Ind. Eng. Chem. Res., 2014, 53(20), 8651-8664.

18 R. P. Swatloski, S. K. Spear, J. D. Holbrey and R. D. Rogers, Dissolution of Cellulose with Ionic Liquids, J. Am. Chem. Soc., 2002, 124(18), 4974-4975.

19 X. F. Sun, Q. Q. Tian, Z. M. Xue, Y. W. Zhang and T. C. Mu, The dissolution behaviour of chitosan in acetate-based ionic liquids and their interactions: from experimental evidence to density functional theory analysis, RSC Adv., 2014, 4(57), 30282-30291.

20 X. F. Sun, Z. M. Xue and T. C. Mu, Precipitation of chitosan from ionic liquid solution by the compressed $\mathrm{CO}_{2}$ antisolvent method, Green Chem., 2014, 16(4), 2102-2106.

21 Z. H. Liu, X. F. Sun, M. Y. Hao, C. Y. Huang, Z. M. Xue and T. C. $\mathrm{Mu}$, Preparation and characterization of regenerated cellulose from ionic liquid using different methods, Carbohydr. Polym., 2015, 117, 99-105.

22 X. F. Sun, C. Y. Huang, Z. M. Xue and T. C. Mu, An Environmentally Benign Cycle To Regenerate Chitosan and Capture Carbon Dioxide by Ionic Liquids, Energy Fuels, 2015, 29(3), 1923-1930.

23 L. S. Chen, W. Zhou and Y. S. Jiang, Determination of the viscosity average molecular weight of chitosan, Chem. Bull., 1996, (4), 57.

24 Y. Ma, L. Xin, H. Tan, M. Fan, J. L. Li, Y. Jia, Z. H. Ling, Y. Chen and X. H. Hu, Chitosan membrane dressings toughened by glycerol to load antibacterial drugs for wound healing, Mater. Sci. Eng., C, 2017, 81, 522.

25 B. Xu, H. Zheng, Y. Wang, Y. An, K. Luo, C. Zhao and W. Xiang, Poly(2-acrylamido-2-methylpropane sulfonic acid) grafted magnetic chitosan microspheres: preparation, characterization and dye adsorption, Int. J. Biol. Macromol., 2018, 112, 648-655.

26 H. R. Kim, J. W. Jang and J. W. Park, Carboxymethyl chitosan-modified magnetic-cored dendrimer as an amphoteric adsorbent, J. Hazard. Mater., 2016, 317, 608-616.

27 D. L. Zhang, L. J. Jiang, J. H. Zong, S. F. Chen, C. Y. Ma and H. J. Li, Incorporated $\alpha$-amylase and starch in an edible chitosan-procyanidin complex film increased the release amount of procyanidins, RSC Adv., 2017, 7(89), 56771-56778.

28 U. J. Kim, H. J. Kim, J. W. Choi, S. Kimura and M. Wada, Cellulose-chitosan beads crosslinked by dialdehyde cellulose, Cellulose, 2017, 24(12), 5517-5528.

29 X. Q. Duan, J. G. Xu, B. H. He, J. R. Li and C. Z. Cheng, Dissolution and regeneration of chitosan in 1-ethyl-3methylimidazolium acetate ionic liquid, New Chem. Mater., 2011, (s1), 56-59.

30 D. D. Xiao, Q. Wang, H. Yan, A. Qin, X. G. Lv, Y. Zhao, M. Zhang, Z. Zhou, J. P. Xu, Q. L. Hu and M. J. Lu, Comparison of morphological and functional restoration between asymmetric bilayer chitosan and bladder acellular matrix graft for bladder augmentation in a rat model, $R S C$ $A d v .$, 2017, 7(67), 42579-42589. 